\title{
STABILITY FOR NEMATIC LIQUID CRYSTALS WITH STRETCHING TERMS
}

\author{
B. CLIMENT-EZQUERRA, F. GUILLÉN-GONZÁLEZ, M. A. \\ RODRÍGUEZ-BELLIDO \\ Department of Ecuaciones Diferenciales y Análisis Numérico, \\ Universidad de Sevilla, Aptdo. 1160, 41080 Sevilla, SPAIN. \\ E-mail:bcliment@us.es,guillen@us.es,angeles@us.es
}

February 27, 2009

Dedicated to the memory of Valery S. Melnik

\begin{abstract}
We study a nematic crystal model appearing in [Liu et al.,2007] modeling stretching effects depending on the different shape of microscopic molecules of the material, under periodic boundary conditions. The aim of the present article is twofold: to extend the results given in [Sun \& Liu, 2009], to a model with more complete stretching terms and to obtain some stability and asymptotic stability properties for this model.
\end{abstract}

Keywords: Nematic Liquid Crystal system, asymptotic stability, stability, stretching effects, existence, regularity.

Mathematics Subject Classifications (2000): 35B65, 35Q35, 76D03

\section{Introduction}

The Nematic Liquid Crystal system is a NavierStokes type model for incompressible fluids respect to the macroscopic variables, that takes into account the crystallinity of the microscopic molecules of the material. It can be obtained coupling NavierStokes equations with the Ginzburg-Landau equations, being its unknowns the solenoidal velocity $\mathbf{u}(t, \mathbf{x})$, the pressure of the fluid $p(t, \mathbf{x})$, and the director field $\mathbf{d}(t, \mathbf{x})$, that represents the orientation of the liquid crystal molecules. Moreover, we suppose that the fluid is confined in a domain $\Omega \subset \mathbb{R}^{3}$.

We deal with an Ericksen-Leslie type formulation. A simplified model was analyzed by F. H. Lin \& C. Liu in [Lin \& Liu, 1995]. In fact, this model is a penalized one depending on the Ginzburg-Landau function:

$$
\mathbf{f}_{\epsilon}(\mathbf{d})=\frac{1}{\epsilon^{2}}\left(|\mathbf{d}|^{2}-1\right) \mathbf{d},
$$

where $|\mathbf{d}|$ denotes the euclidean norm in $\mathbb{R}^{3}$ and $\epsilon>0$ is a penalization parameter. This penalization function has a potential structure, i. e. there exists the function $F_{\epsilon}(\mathbf{d})=\frac{1}{4 \epsilon^{2}}\left(|\mathbf{d}|^{2}-1\right)^{2}$ such that $\mathbf{f}_{\epsilon}(\mathbf{d})=\nabla_{\mathbf{d}}\left(F_{\epsilon}(\mathbf{d})\right)$ for all $\mathbf{d} \in \mathbb{R}^{3}$.

We denote $Q=(0,+\infty) \times \Omega$ and $\Sigma=(0,+\infty) \times$ $\partial \Omega$, where $\Omega \subset \mathbb{R}^{3}$ is a smooth enough domain and $\partial \Omega$ its boundary. We consider the EDP system appearing in [Liu et al.,2007; system (1.9), p. 1187], that reads as:

$(L C)\left\{\begin{aligned} D_{t} \mathbf{u}-\nu \Delta \mathbf{u}+\nabla p-\lambda \nabla \cdot \sigma^{e} & =\mathbf{0} \text { in } Q, \\ \nabla \cdot \mathbf{u} & =0 \text { in } Q, \\ \mathcal{D}_{t} \mathbf{d}+\gamma \mathbf{w} & =\mathbf{0} \text { in } Q,\end{aligned}\right.$ 
B. Climent-Ezquerra et al.

being

$$
D_{t} \mathbf{u}=\partial_{t} \mathbf{u}+(\mathbf{u} \cdot \nabla) \mathbf{u}
$$

the material derivative of $\mathbf{u}$,

$$
\sigma^{e}=-(\nabla \mathbf{d})^{t} \nabla \mathbf{d}-\beta \mathbf{w} \mathbf{d}^{t}-(1+\beta) \mathbf{d} \mathbf{w}^{t}
$$

the elastic stress tensor $(\beta \in \mathbb{R})$ and

$$
\mathbf{w}=-\Delta \mathbf{d}+\mathbf{f}_{\epsilon}(\mathbf{d})
$$

the Euler-Lagrange system derived from the minimization problem respect to the elastic energy

$$
E_{e}(\mathbf{d})=\frac{1}{2} \int_{\Omega}|\nabla \mathbf{d}|^{2}+\int_{\Omega} \mathbf{F}_{\epsilon}(\mathbf{d}) .
$$

The term

$$
\mathcal{D}_{t} \mathbf{d}=D_{t} \mathbf{d}+C(\mathbf{d}, \nabla \mathbf{u})
$$

describes a general derivative containing the material derivative $D_{t} \mathbf{d}=\partial_{t} \mathbf{d}+(\mathbf{u} \cdot \nabla) \mathbf{d}$ and the quadratic term

$$
C(\mathbf{d}, \nabla \mathbf{u})=\beta(\nabla \mathbf{u}) \mathbf{d}+(1+\beta)(\nabla \mathbf{u})^{t} \mathbf{d}
$$

modeling the so-called stretching effects, depending on the form of the molecules [Liu et al., 2007]. In fact, the constant $\beta=-\alpha$ is associated with the aspect ratio $r$ of the ellipsoid particles. The case of $\alpha$ near to 1 corresponds to rod like particles (then the transport is purely covariant stretching), the case of $\alpha$ near to 0 corresponds to disc like particles (then the transport is anti-stretching) and the case of $\alpha$ near to $1 / 2$ corresponds to the spherical shape (the transport is the rigid rotation of the center of the mass).

Finally, $\nu>0$ is the fluid viscosity, $\lambda>0$ is the elasticity constant and $\gamma>0$ is a relaxation in time constant.

The theoretical analysis of a simplified model without stretching effects, i.e for $C(\mathbf{d}, \nabla \mathbf{u})=0$ and the corresponding elastic tensor $\sigma^{e}=-(\nabla \mathbf{d})^{t} \nabla \mathbf{d}$, was made in [Lin \& Liu, 1995] obtaining existence of global weak solution, i. e.

$$
\begin{aligned}
& \mathbf{u} \in L^{\infty}\left(0, T ; \mathbf{L}^{2}(\Omega)\right) \cap L^{2}\left(0, T ; \mathbf{H}^{1}(\Omega)\right), \\
& \mathbf{d} \in L^{\infty}\left(0, T ; \mathbf{H}^{2}(\Omega)\right) \cap L^{2}\left(0, T ; \mathbf{H}^{3}(\Omega)\right),
\end{aligned}
$$

for all $T>0$, and the existence (and uniqueness) of local strong solution, i. e.

$$
\begin{aligned}
& \mathbf{u} \in L^{\infty}\left(0, T_{*} ; \mathbf{H}^{1}(\Omega)\right) \cap L^{2}\left(0, T_{*} ; \mathbf{H}^{2}(\Omega)\right), \\
& \mathbf{d} \in L^{\infty}\left(0, T_{*} ; \mathbf{H}^{2}(\Omega)\right) \cap L^{2}\left(0, T_{*} ; \mathbf{H}^{3}(\Omega)\right),
\end{aligned}
$$

with $T_{*} \leq T$ (small enough) or $T_{*}=T$ (for each $T>0$ ) for big enough viscosity coefficient $\nu$ or for two-dimensional domains. All these previous results are given for the time-independent Dirichlet boundary data:

$$
\mathbf{u}=\mathbf{0}, \quad \mathbf{d}=\mathbf{h} \quad \text { on } \Sigma, \quad(\mathbf{h} \neq \mathbf{h}(t))
$$

and for the initial-value boundary problem with initial condition:

$$
\left.\mathbf{u}\right|_{t=0}=\mathbf{u}_{0},\left.\quad \mathbf{d}\right|_{t=0}=\mathbf{d}_{0} \quad \text { in } \Omega .
$$

When time-dependent Dirichlet data for $\mathbf{d}$ is considered $(\mathbf{h}=\mathbf{h}(t))$, the existence of weak timeperiodic solution, that is solutions obtained by changing (3) by $\mathbf{u}(0)=\mathbf{u}(T)$ and $\mathbf{d}(\mathbf{0})=$ $\mathbf{d}(T)$, is obtained in [Climent-Ezquerra et al.,]. The strong regularity up to infinite time for big enough viscosity $\nu$ jointly with the strong regularity of time-periodic solutions are obtained in [Climent-Ezquerra et al.,].

The results corresponding to the initialvalue boundary problem are extended in [Lin \& Liu, 2000] to a much more complete model respect to the dissipative tensor and considering the particular stretching effects for the case of spherical molecules, i.e. taking $\beta=-1 / 2$ in (1).

Recently, a liquid crystal model with a stretching term for the case of rod like particles (taking $\beta=-1$ in (1)) and periodic boundary conditions for both $\mathbf{u}$ and $\mathbf{d}$ has been studied in [Sun \& Liu, 2009], obtaining global weak solution and local strong solution (which is global for large enough viscosity)

The aim of the present article is twofold: to extend the last results of [Sun \& Liu, 2009] to a model with more complete stretching terms and to obtain some stability and asymptotic stability properties for this model.

\section{General Framework.}

Assume that we have the following situation, a.e. $t \in\left(t_{0},+\infty\right):$

$$
E(t), F(t) \geq 0, \quad E^{\prime}(t)+F(t) \leq 0 .
$$

Then, $E \in C_{b}\left[t_{0},+\infty\right)$, is a decreasing function and there exists

$$
\lim _{t \rightarrow+\infty} E(t)=E_{\infty} \geq 0
$$


On the other hand, $F \in L^{1}\left(t_{0},+\infty\right)$, that is,

$$
\int_{t_{0}}^{+\infty} F(t) d t<+\infty
$$

In this case, for any $\delta>0$, there exists a large enough time $t_{1}^{*}=t_{1}^{*}(\delta) \geq t_{0}$ such that:

$$
\int_{t_{1}^{*}}^{+\infty} F(t) d t \leq \delta .
$$

In particular, we can say that for each $\delta>0$ there exists a large enough time $t_{1}^{*}(\delta) \geq t_{0}$ such that

$$
\frac{1}{\tau} \int_{t}^{t+\tau} F(t) d t \leq \frac{\delta}{\tau}, \quad \forall \tau>0, \forall t \geq t_{1}^{*}(\delta) .
$$

Lemma 2.1. Let $F \in L^{1}\left(t_{0},+\infty\right), F \geq 0$ in $\left(t_{0},+\infty\right)$, satisfying (6). Then, $\forall \delta>0, \forall t \geq t_{1}^{*}(\delta)$ and $\forall \tau>0$ there exists a time $\bar{t} \in[t, t+\tau]$ such that:

$$
F(\bar{t}) \leq \frac{2 \delta}{\tau}
$$

Indeed, the set of points $\bar{t} \in[t, t+\tau]$ satisfying (7) has measure $\geq \tau / 2$.

Proof. We focus on the proof in the interval $\left[t_{1}^{*}, t_{1}^{*}+\right.$ $\tau$ ]. The proof for another interval of length $\tau$ contained in $\left[t_{1}^{*},+\infty\right)$ is similar.

Indeed, we define:

$$
A=\left\{s \in\left[t_{1}^{*}, t_{1}^{*}+\tau\right] / F(s) \geq \frac{2 \delta}{\tau}\right\} .
$$

Therefore,

$$
\int_{A} F(t) d t+\int_{A^{c}} F(t) d t \leq \delta
$$

and thus

$$
\frac{2 \delta}{\tau}|A| \leq \delta \quad \Rightarrow \quad|A| \leq \frac{\tau}{2} .
$$

That is, $\left|A^{c}\right| \geq \tau / 2$.

Now, we assume that the following differential inequality for $F(t)$ holds:

$$
F^{\prime}(t) \leq C_{2}\left(F(t)^{3}+1\right) .
$$

Lemma 2.2. Let $F \in L^{1}\left(t_{0},+\infty\right)$ be a function satisfying the differential inequality (8). For any $\varepsilon<1$, if $F\left(t_{0}\right) \leq \varepsilon / 3$, then $F(t) \leq \varepsilon \forall t \in$ $\left[t_{0}, t_{0}+T_{*}(\varepsilon)\right]$, where $T_{*}(\varepsilon)=\frac{\varepsilon}{3 C_{2}}$.
Proof. We argue by contradiction: Suppose that there exists a time $t_{1} \in\left[t_{0}, t_{0}+T_{*}(\varepsilon)\right]$ such that $F(t)<\varepsilon$ in $\left[t_{0}, t_{1}\right)$ and $F\left(t_{1}\right)=\varepsilon$. Then, from eq. (8) we obtain that $F^{\prime}<2 C_{2}$ en $\left[t_{0}, t_{1}\right]$. Integrating in $\left[t_{0}, t_{1}\right]$, we get:

$$
\begin{aligned}
F\left(t_{1}\right) & <F\left(t_{0}\right)+2 C_{2}\left(t_{1}-t_{0}\right) \\
& \leq F\left(t_{0}\right)+2 C_{2} T_{*}(\varepsilon) \\
& \leq \frac{\varepsilon}{3}+2 C_{2} \frac{\varepsilon}{3 C_{2}}=\varepsilon .
\end{aligned}
$$

This fact contradicts the starting hypothesis.

\subsection{Asymptotic stability.}

Theorem 2.3. Let $\varepsilon<1$, and $F \in L^{1}(0,+\infty)$, $F \geq 0$, such that both (8) and inequality (6) hold for $\delta=\frac{\varepsilon^{2}}{36 C_{2}}, t_{1}^{*}=t_{1}^{*}(\delta)$ and $\tau=\frac{T_{*}(\varepsilon)}{2}$. Then,

$$
F(t) \leq \varepsilon, \forall t \geq t_{2}^{*}=t_{1}^{*}+\frac{T_{*}(\varepsilon)}{2}=t_{1}^{*}(\delta)+\frac{\varepsilon}{6 C_{2}}
$$

Remark 2.4. In particular, $F \in W^{1,1}\left(t_{2}^{*},+\infty\right) \hookrightarrow$ $C\left[t_{2}^{*},+\infty\right)$.

Proof. We argue by contradiction: Assume that there exists a time $\widetilde{t}>t_{2}^{*}$ such that $F(t)>\varepsilon$. We consider the interval $\left[\widetilde{t}-T_{*}(\varepsilon) / 2, \widetilde{t}\right] \subset\left[t_{1}^{*},+\infty\right)$.

From Lemma 2.1 we conclude that for each interval of length $T_{*}(\varepsilon) / 2$ contained in $\left[t_{1}^{*},+\infty\right)$ and $\forall t \geq t_{1}^{*}$ there exists a time $\bar{t}_{1} \in\left[\widetilde{t}-T_{*}(\varepsilon) / 2, \widetilde{t}\right]$ such that:

$$
F\left(\bar{t}_{1}\right) \leq \frac{2 \delta}{\tau}=\frac{\varepsilon^{2} /\left(18 C_{2}\right)}{\varepsilon /\left(6 C_{2}\right)}=\frac{\varepsilon}{3} .
$$

Thus, applying Lemma 2.2, one verifies:

$$
F(t) \leq \varepsilon, \quad \forall t \in\left[\bar{t}_{1}, \bar{t}_{1}+T_{*}(\varepsilon)\right] .
$$

Observe that $\widetilde{t} \in\left[\bar{t}_{1}, \bar{t}_{1}+T_{*}(\varepsilon)\right]$, which gives us to contradiction.

Corollary 2.5. Let $F \in L^{1}\left(t_{0},+\infty\right)$ be a function satisfying eq. (8). Then, $F(t)$ is a function asymptotically stable to 0 , that is,

$$
\lim _{t \rightarrow+\infty} F(t)=0
$$




\subsection{Stability until infinite time.}

If we assume that:

$$
E\left(t_{0}\right) \leq \delta(\varepsilon)=\frac{\varepsilon^{2}}{36 C_{2}},
$$

then, from (4) we get: $\forall t_{1}>t_{0}$

$$
\int_{t_{0}}^{t_{1}} F(t) d t \leq E\left(t_{0}\right)-E\left(t_{1}\right) \leq \delta(\varepsilon)
$$

In fact, one has (5) for $t_{1}^{*}(\varepsilon)=t_{0}$. Then, applying Theorem 2.3, we obtain:

$$
F(t) \leq \varepsilon \quad \forall t \geq t_{0}+\frac{\varepsilon}{6 C_{2}}\left(=t_{0}+\frac{T_{*}(\varepsilon)}{2}\right)
$$

If, moreover,

$$
F\left(t_{0}\right) \leq \frac{\varepsilon}{3},
$$

then applying Lemma 2.2, we get:

$$
F(t) \leq \varepsilon \quad \forall t \in\left[t_{0}, t_{0}+T_{*}(\varepsilon)\right]
$$

In summary, assuming $(H 1)$ and $(H 2)$, one has:

$$
E(t) \leq \delta(\varepsilon), \quad F(t) \leq \varepsilon, \quad \forall t \geq t_{0} .
$$

\section{The Nematic Liquid Crystal Model}

\subsection{Weak estimates}

If we consider both $\mathbf{u}(t)$ and $\mathbf{w}(t)$ as test functions in the $\mathbf{u}$-system and $\mathbf{d}$-system of $(L C)$ respectively, taking into account the equality:

$$
\nabla \cdot\left((\nabla \mathbf{d})^{t} \nabla \mathbf{d}\right)=-(\nabla \mathbf{d})^{t} \mathbf{w}+\nabla E_{e}(\mathbf{d}),
$$

we obtain:

$$
\begin{aligned}
& \frac{1}{2} \frac{d}{d t}\|\mathbf{u}(t)\|_{\mathbf{L}^{2}(\Omega)}^{2}+\nu\|\nabla \mathbf{u}(t)\|_{\mathbf{L}^{2}(\Omega)}^{2} \\
& +-\lambda \int_{\Omega}[(\mathbf{u} \cdot \nabla) \mathbf{d}] \cdot \mathbf{w} d \mathbf{x} \\
& -\lambda \int_{\Omega}\left[\beta \mathbf{w}^{t} \mathbf{d}+(1+\beta) \mathbf{d}^{t} \mathbf{w}\right]: \nabla \mathbf{u} d \mathbf{x}=0
\end{aligned}
$$

and

$$
\begin{array}{r}
\frac{d}{d t}\left(\frac{1}{2}\|\nabla \mathbf{d}(t)\|_{\mathbf{L}^{2}(\Omega)}^{2}+F_{\epsilon}(\mathbf{d})(t)\right)+\gamma\|\mathbf{w}\|_{\mathbf{L}^{2}(\Omega)}^{2} \\
+\int_{\Omega}[(\mathbf{u} \cdot \nabla) \mathbf{d}] \cdot \mathbf{w} d \mathbf{x}+\int_{\Omega} C(\mathbf{d}, \nabla \mathbf{u}) \cdot \mathbf{w} d \mathbf{x}=0
\end{array}
$$

for any boundary conditions for $(\mathbf{u}, \mathbf{d})$ given in the Introduction (that is, Dirichlet, Neumann or periodic for $\mathbf{d}$ ). Then, adding (12) to (13) multiplied by $\lambda$, the last two terms of (12) and (13) cancel and the so-called energy equality holds:

$$
\begin{aligned}
& \frac{d}{d t}\left[\frac{1}{2}\|\mathbf{u}(t)\|_{\mathbf{L}^{2}(\Omega)}^{2}+\lambda E_{e}(\mathbf{d}(t))\right] \\
& +\nu\|\nabla \mathbf{u}(t)\|_{\mathbf{L}^{2}(\Omega)}^{2}+\lambda \gamma\|\mathbf{w}(t)\|_{\mathbf{L}^{2}(\Omega)}^{2}=0
\end{aligned}
$$

(recall that $E_{e}(\mathbf{d})$ is given in $(2)$ ).

Note that, defining the time functions $E(t)$ and $F(t)$ as:

$$
\begin{aligned}
E(t) & =\frac{1}{2}\|\mathbf{u}(t)\|_{\mathbf{L}^{2}(\Omega)}^{2}+\lambda E_{e}(\mathbf{d}(t)) \\
F(t) & =\nu\|\nabla \mathbf{u}(t)\|_{\mathbf{L}^{2}(\Omega)}^{2}+\gamma \lambda\|\mathbf{w}(t)\|_{\mathbf{L}^{2}(\Omega)}^{2}
\end{aligned}
$$

the inequality (4) is deduced from (14). As a consequence, we can deduce the existence of weak solutions of the problem. Moreover, by using this weak regularity and the $\mathbf{H}^{2}(\Omega)$ and $\mathbf{H}^{3}(\Omega)$ regularity of the elliptic problem $-\Delta \mathbf{d}+f_{\epsilon}(\mathbf{d})=\mathbf{w}$ with appropriate boundary conditions, we can deduce [Climent-Ezquerra et al.,]:

$$
\begin{aligned}
\|\mathbf{d}\|_{\mathbf{H}^{2}(\Omega)} & \leq C\left(\|\mathbf{w}\|_{\mathbf{L}^{2}(\Omega)}+1\right), \\
\|\mathbf{d}\|_{\mathbf{H}^{3}(\Omega)} & \leq C\left(\|\mathbf{w}\|_{\mathbf{H}^{1}(\Omega)}+1\right) .
\end{aligned}
$$

In the next section, we will use repeatedly these estimates.

\subsection{Strong estimates}

In this section, we only consider the periodic boundary conditions case for all variables $(\mathbf{u}, p, \mathbf{d})$. Taking both $-\Delta \mathbf{u}$ and $-\lambda \Delta \mathbf{w}$ as test functions in the $\mathbf{u}$-system and in the $\mathbf{d}$-system of $(L C)$ respectively, one can obtain ([Sun \& Liu, 2009]):

$$
\begin{aligned}
& \frac{d}{d t}\|\nabla \mathbf{u}\|_{\mathbf{L}^{2}(\Omega)}^{2}+\nu\|\Delta \mathbf{u}\|_{\mathbf{L}^{2}(\Omega)}^{2} \\
& \leq\|(\mathbf{u} \cdot \nabla) \mathbf{u}\|_{\mathbf{L}^{2}(\Omega)}^{2}+C \frac{\lambda^{2}}{\nu}\left\|(\nabla \mathbf{d})^{t} \mathbf{w}\right\|_{\mathbf{L}^{2}(\Omega)}^{2} \\
& \quad+\lambda \int_{\Omega}\left\{\beta \mathbf{w} \mathbf{d}^{t}+(1+\beta) \mathbf{d w}^{t}\right\} \nabla(\Delta \mathbf{u}) d \mathbf{x}
\end{aligned}
$$


and

$$
\begin{gathered}
\lambda \int_{\Omega} \nabla \partial_{t} \mathbf{d}: \nabla \mathbf{w} d \mathbf{x}+\lambda \gamma\|\nabla \mathbf{w}\|_{\mathbf{L}^{2}(\Omega)}^{2} \\
+\lambda \int_{\Omega} \nabla((\mathbf{u} \cdot \nabla) \mathbf{d}): \nabla \mathbf{w} d \mathbf{x} \\
\quad+\lambda \int_{\Omega} \nabla C(\mathbf{d}, \nabla \mathbf{u}): \nabla \mathbf{w} d \mathbf{x}=0
\end{gathered}
$$

Observe that the first term of (19) can be rewritten as:

$$
\begin{aligned}
& \int_{\Omega} \nabla \partial_{t} \mathbf{d}: \nabla \mathbf{w} d \mathbf{x}=-\int_{\Omega} \Delta\left(\partial_{t} \mathbf{d}\right) \mathbf{w} d \mathbf{x} \\
& =\int_{\Omega} \partial_{t} \mathbf{w} \mathbf{w} d \mathbf{x}-\int_{\Omega} f_{\epsilon}^{\prime}(\mathbf{d})\left(\partial_{t} \mathbf{d}\right) \mathbf{w} d \mathbf{x} \\
& =\frac{1}{2} \frac{d}{d t}\|\mathbf{w}\|_{\mathbf{L}^{2}(\Omega)}^{2}-\int_{\Omega} f_{\epsilon}^{\prime}(\mathbf{d})\left(\partial_{t} \mathbf{d}\right) \mathbf{w} d \mathbf{x}
\end{aligned}
$$

Now, using the $\mathbf{d}$-system of $(L C)$, that is, $\partial_{t} \mathbf{d}=$ $-(\mathbf{u} \cdot \nabla) \mathbf{d}-C(\mathbf{d}, \nabla \mathbf{u})-\gamma \mathbf{w}$, one has:

$$
\begin{aligned}
& -\int_{\Omega} f_{\epsilon}^{\prime}(\mathbf{d})\left(\partial_{t} \mathbf{d}\right) \mathbf{w} d \mathbf{x} \\
& =\int_{\Omega} f_{\epsilon}^{\prime}(\mathbf{d})((\mathbf{u} \cdot \nabla) \mathbf{d}+C(\mathbf{d}, \nabla \mathbf{u})+\gamma \mathbf{w}) \mathbf{w} d \mathbf{x} \\
& \leq \varepsilon\left(\|\Delta \mathbf{u}\|_{\mathbf{L}^{2}(\Omega)}^{2}+\|\nabla \mathbf{w}\|_{\mathbf{L}^{2}(\Omega)}^{2}\right) \\
& +C_{\varepsilon}\left(F^{3}(t)+1\right)
\end{aligned}
$$

for $\varepsilon$ small enough.

The more nonlinear terms of the last term of (19) are manipulated as follows (here, the periodic boundary conditions are again applied):

$$
\begin{aligned}
& \lambda \int_{\Omega}\left(\beta \nabla(\nabla \mathbf{u}) \mathbf{d}+(1+\beta) \nabla(\nabla \mathbf{u})^{t} \mathbf{d}\right): \nabla \mathbf{w} d \mathbf{x} \\
& \quad \leq-\lambda \int_{\Omega} \nabla(\Delta \mathbf{u}):\left\{\beta \mathbf{w}^{t}+(1+\beta) \mathbf{d} \mathbf{w}^{t}\right\} d \mathbf{x} \\
& \quad+C\left\|D^{2} \mathbf{u}\right\|_{\mathbf{L}^{2}(\Omega)}\|\nabla \mathbf{d}\|_{\mathbf{L}^{6}(\Omega)}\|\mathbf{w}\|_{\mathbf{L}^{3}(\Omega)}
\end{aligned}
$$

Note that the first term on the right-hand side of (22) cancels with the last term in (18). The remaining part of the last term of (19) can be written as:

$$
\begin{aligned}
& \lambda \int_{\Omega}(\beta(\nabla \mathbf{d} \cdot \nabla) \mathbf{u}: \nabla \mathbf{w} \\
& +(1+\beta)(\nabla \mathbf{w} \cdot \nabla) \mathbf{u}: \nabla \mathbf{d}) d \mathbf{x} \\
& \leq C(\lambda, \beta)\|\nabla \mathbf{w}\|_{\mathbf{L}^{2}(\Omega)}\|\nabla \mathbf{d}\|_{\mathbf{L}^{6}(\Omega)}\|\nabla \mathbf{u}\|_{\mathbf{L}^{3}(\Omega)}
\end{aligned}
$$

being $C(\lambda, \beta)$ a constant depending on $\lambda$ and $\beta$.

Therefore, adding (18) to (19) and taking into account estimates (20)-(23), we obtain:

$$
\begin{aligned}
& \frac{d}{d t}\left(\|\nabla \mathbf{u}\|_{\mathbf{L}^{2}(\Omega)}^{2}+\lambda\|\mathbf{w}\|_{\mathbf{L}^{2}(\Omega)}^{2}\right) \\
& +\nu\|\Delta \mathbf{u}\|_{\mathbf{L}^{2}(\Omega)}^{2}+\lambda \gamma\|\nabla \mathbf{w}\|_{\mathbf{L}^{2}(\Omega)}^{2} \\
& \leq\|(\mathbf{u} \cdot \nabla) \mathbf{u}\|_{\mathbf{L}^{2}(\Omega)}^{2}+C \frac{\lambda^{2}}{\nu}\left\|(\nabla \mathbf{d})^{t} \mathbf{w}\right\|_{\mathbf{L}^{2}(\Omega)}^{2} \\
& +\lambda \int_{\Omega} \nabla((\mathbf{u} \cdot \nabla) \mathbf{d}): \nabla \mathbf{w} d \mathbf{x} \\
& +C\left(F^{3}(t)+1\right) \\
& +C\left\|D^{2} \mathbf{u}\right\|_{\mathbf{L}^{2}(\Omega)}\|\nabla \mathbf{d}\|_{\mathbf{L}^{6}(\Omega)}\|\mathbf{w}\|_{\mathbf{L}^{3}(\Omega)} \\
& +C(\lambda, \beta)\|\nabla \mathbf{w}\|_{\mathbf{L}^{2}(\Omega)}\|\nabla \mathbf{d}\|_{\mathbf{L}^{6}(\Omega)}\|\nabla \mathbf{u}\|_{\mathbf{L}^{3}(\Omega)} \\
& =\sum_{i=1}^{6} I_{i}
\end{aligned}
$$

Using Sobolev's inequalities, the estimates for the $I_{i}$-terms can be summarized as follows:

$$
\begin{gathered}
I_{1} \leq\|\mathbf{u}\|_{\mathbf{L}^{6}(\Omega)}^{2}\|\nabla \mathbf{u}\|_{\mathbf{L}^{3}(\Omega)}^{2} \\
\leq \varepsilon\|\Delta \mathbf{u}\|_{\mathbf{L}^{2}(\Omega)}^{2}+C_{\varepsilon}\|\nabla \mathbf{u}\|_{\mathbf{L}^{2}(\Omega)}^{6} \\
I_{2} \leq\|\nabla \mathbf{d}\|_{\mathbf{L}^{6}(\Omega)}^{2}\|\mathbf{w}\|_{\mathbf{L}^{3}(\Omega)}^{2} \\
\leq \varepsilon\|\nabla \mathbf{w}\|_{\mathbf{L}^{2}(\Omega)}^{2}+C_{\varepsilon}\|\mathbf{w}\|_{\mathbf{L}^{2}(\Omega)}^{6} \\
I_{3} \leq\|\nabla \mathbf{u}\|_{\mathbf{L}^{3}(\Omega)}\|\nabla \mathbf{d}\|_{\mathbf{L}^{6}(\Omega)}\|\nabla \mathbf{w}\|_{\mathbf{L}^{2}(\Omega)} \\
+\|\mathbf{u}\|_{\mathbf{L}^{6}(\Omega)}\|\Delta \mathbf{d}\|_{\mathbf{L}^{3}(\Omega)}\|\nabla \mathbf{w}\|_{\mathbf{L}^{2}(\Omega)}=I_{31}+I_{32}
\end{gathered}
$$

where term $I_{31}$ as the same type of estimates as term $I_{6}$.

$$
\begin{gathered}
I_{32} \leq\|\mathbf{u}\|_{\mathbf{L}^{6}(\Omega)}\|\Delta \mathbf{d}\|_{\mathbf{L}^{3}(\Omega)}\|\nabla \mathbf{w}\|_{\mathbf{L}^{2}(\Omega)} \\
\leq \varepsilon\|\nabla \mathbf{w}\|_{\mathbf{L}^{2}(\Omega)}+C_{\varepsilon}\|\nabla \mathbf{u}\|_{\mathbf{L}^{2}(\Omega)}^{4}\|\mathbf{w}\|_{\mathbf{L}^{2}(\Omega)}^{2} \\
I_{5} \leq \varepsilon\left(\|\Delta \mathbf{u}\|_{\mathbf{L}^{2}(\Omega)}^{2}+\|\nabla \mathbf{w}\|_{\mathbf{L}^{2}(\Omega)}^{2}\right)+C_{\varepsilon}\|\mathbf{w}\|_{\mathbf{L}^{2}(\Omega)}^{6} \\
I_{6} \leq \varepsilon\left(\|\Delta \mathbf{u}\|_{\mathbf{L}^{2}(\Omega)}^{2}+\|\nabla \mathbf{w}\|_{\mathbf{L}^{2}(\Omega)}^{2}\right) \\
+C_{\varepsilon}\|\mathbf{w}\|_{\mathbf{L}^{2}(\Omega)}^{4}\|\nabla \mathbf{u}\|_{\mathbf{L}^{2}(\Omega)}^{2}
\end{gathered}
$$

Now, we introduce function $G(t)$ defined as:

$$
G(t)=\nu\|\Delta \mathbf{u}\|_{\mathbf{L}^{2}(\Omega)}^{2}+\lambda \gamma\|\nabla \mathbf{w}\|_{\mathbf{L}^{2}(\Omega)}^{2}
$$

Observe that, finally, (24) can be written in the following form:

$$
F^{\prime}(t)+G(t) \leq C\left(1+F^{3}(t)\right)
$$


being $F(t)$ and $G(t)$ the functions defined in (16) and (25) respectively. Therefore, function $F(t)$ satisfies (8).

\section{Applications of the General Framework to Nematic Liquid Crystal.}

\subsection{Asymptotic stability.}

Let $\left(\mathbf{u}_{0}, \mathbf{d}_{0}\right) \in \mathbf{H}^{1}(\Omega) \times \mathbf{H}^{2}(\Omega)$ be two given functions and $(\mathbf{u}(t), \mathbf{d}(t))$ a weak solution of system $(L C)$ with periodic boundary conditions and initial data $\left(\mathbf{u}_{0}, \mathbf{d}_{0}\right)$.

Since (4) and (8) hold, applying the results from Section 2, one has:

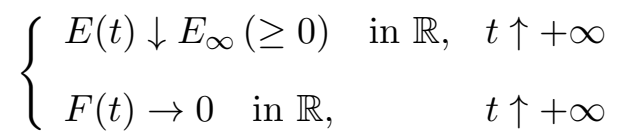

hence:

$$
\left\{\begin{array}{lll}
E^{\prime}(t) \rightarrow 0 & \text { in } \mathbb{R} & t \uparrow+\infty \\
\mathbf{u}(t) \rightarrow 0 & \text { in } \mathbf{H}_{0}^{1}(\Omega) & t \uparrow+\infty \\
\mathbf{w}(t) \rightarrow 0 & \text { in } \mathbf{L}^{2}(\Omega) & t \uparrow+\infty
\end{array}\right.
$$

Moreover, for each subsequence $t_{j} \uparrow+\infty$, there exists a subsequence $\left(t_{j_{k}}\right) \subset\left(t_{j}\right)$ such that:

$$
\mathbf{d}\left(t_{j k}\right) \rightarrow \overline{\mathbf{d}} \quad \text { in } \mathbf{H}^{2}(\Omega) \text {-weak } \text { for } k \uparrow+\infty .
$$

being $\overline{\mathbf{d}}$ a critical point of the elastic energy $E_{e}(\mathbf{d})$, that is, a solution for the stationary problem:

$$
-\Delta \overline{\mathbf{d}}+f_{\varepsilon}(\overline{\mathbf{d}})=\mathbf{0} \text { in } \Omega,
$$

with periodic boundary conditions on $\partial \Omega$. Note that,

$$
E_{\infty}=\frac{\lambda}{2}\left(|\nabla \overline{\mathbf{d}}(t)|_{2}^{2}+2 \int_{\Omega} F(\overline{\mathbf{d}}(t))\right)=\lambda E_{e}(\overline{\mathbf{d}})
$$

that is, every possible limit of the director field $\overline{\mathbf{d}}$ when $t \uparrow+\infty$ is a critical point of the elastic energy and all these possible limits have the same elastic energy $E_{\infty}$.

\subsection{Stability for constant director fields.}

If $\left(\mathbf{u}_{0}, \mathbf{d}_{0}\right)$ are such that:

$(H 1)$

$$
\frac{1}{2}\left\|\mathbf{u}_{0}\right\|_{\mathbf{L}^{2}(\Omega)}^{2}+\frac{\lambda}{2}\left\|\nabla \mathbf{d}_{0}\right\|_{\mathbf{L}^{2}(\Omega)}^{2}+\lambda \int_{\Omega} F_{\epsilon}\left(\mathbf{d}_{0}\right) \leq \delta(\varepsilon)
$$

(in particular, $\left\|\mathbf{d}_{c t e}-\mathbf{d}_{0}\right\|_{\mathbf{H}^{1}(\Omega)}^{2} \leq C \varepsilon^{2}$ for a constant vector $\overline{\mathbf{d}}_{\text {cte }}$ with $\left.\left|\overline{\mathbf{d}}_{c t e}\right|=1\right)$ and

$$
\nu\left\|\nabla \mathbf{u}_{0}\right\|_{\mathbf{L}^{2}(\Omega)}^{2}+\gamma \lambda\left\|\mathbf{w}_{0}\right\|_{\mathbf{L}^{2}(\Omega)}^{2} \leq \frac{\varepsilon}{3},
$$

where $\mathbf{w}_{0}=-\Delta \mathbf{d}_{0}+\mathbf{f}_{\epsilon}\left(\mathbf{d}_{0}\right)$ then for each $t \geq t_{0}$, applying the results from Section 2 one has:

$\frac{1}{2}\|\mathbf{u}(t)\|_{\mathbf{L}^{2}(\Omega)}^{2}+\frac{\lambda}{2}\|\nabla \mathbf{d}(t)\|_{\mathbf{L}^{2}(\Omega)}^{2}+\lambda \int_{\Omega} F_{\epsilon}(\mathbf{d}(t)) \leq \delta(\varepsilon)$

and

$$
\nu\|\nabla \mathbf{u}(t)\|_{\mathbf{L}^{2}(\Omega)}^{2}+\gamma \lambda\|\mathbf{w}(t)\|_{\mathbf{L}^{2}(\Omega)}^{2} \leq \frac{\varepsilon}{3} .
$$

\section{Acknowledgements}

The authors have been partially supported by Projects MTM2006-07932 and P06-FQM-02373.

\section{References}

Climent-Ezquerra, B., Guillén-González, F. \& Rojas-Medar, M.A. [2006] "Reproductivity for a nematic liquid crystal model," Z. Angew. Math. Phys. 576, No. 6, 984-998.

Climent-Ezquerra, B., Guillén-González, F. \& Moreno-Iraberte, M. J. [] "Regularity and Time-periodicity for a Nematic Liquid Crystal model," Accepted in Nonlinear Analysis , http://dx.doi.org/10.1016/j.na.2008.10.092.

Lin, F. H.\& Liu, C. [1995] "Nonparabolic Dissipative Systems Modeling the Flow of Liquid Crystals," Comm. Pure Appl. Math. 48, 501-537.

Lin, F. H. \& Liu, C. [2000] "Existence of Solutions for the Ericksen-Leslie System," Arch. Rational Mech. Anal. 154, 135-156.

Liu, C., Shen, J. \& Yang, X. [2007] "Dynamics of Defect Motion in Nematic Liquid Crystal Flow: Modeling and Numerical Simulation," Commun. Comput. Phys. 2-No. 6, 1184-1198.

Sun, H. \& Liu, C. [2009] "On Energetic Variational Approaches in Modeling the Nematic Liquid Crystal Flows," Discrete and Continuous Dynamical Systems 23-No.1\&2, 455-475. 